\title{
El reporte radiológico en pacientes con sospecha de COVID-19: nuestra experiencia en el Centro Médico ABC
}

\section{The radiological report in patients with suspected COVID-19:}

\section{our experience at $A B C$ Medical Center}

Estefanía Murrieta-Peralta, Ana P. Chischistz-Condey, Jorge Ramírez-Landero,

Carla R. Moctezuma-Velasco*, Héctor Murrieta-González y Jonathan Salazar-Segovia

Servicio de Imagenología Diagnóstica y Terapéutica, Departamento de Radiología, Centro Médico ABC, Ciudad de México, México

\section{RESUMEN}

Introducción: Ante la pandemia de la enfermedad por coronavirus 2019 (COVID-19), la tomografía (TC) ha mostrado ser útil en estadios tempranos, ya que los hallazgos pueden presentarse antes del inicio de los síntomas. Publicaciones recientes describen hallazgos típicos y atípicos en TC que podemos encontrar en pacientes con COVID-19. Diversas sociedades de radiología a nivel internacional han propuesto sistemas de clasificación para determinar el grado de sospecha con base en estos hallazgos, así como realizar informes estructurados. Objetivo: En el Centro Médico ABC ha surgido la necesidad de implementar un reporte estandarizado que facilite la integración de los hallazgos observados, disminuya la variabilidad interobservador y mejore la comunicación con los médicos tratantes para permitir la toma de decisiones clínicas oportunas. Material y métodos: Revisamos los sistemas de clasificación que existen en la literatura para la categorización de hallazgos tomográficos de COVID-19, centrados principalmente en los que pretenden dirigir el tratamiento de los casos sospechosos. Resultados: La consistencia de los hallazgos observados por radiólogos de distintas nacionalidades y experiencia hace que la categorización propuesta en consenso por la Sociedad Radiológica de Norteamérica, el Colegio Americano de Radiología y la Sociedad Torácica de Radiología sea fácil de aplicar y 
reproducible. Conclusiones: Se decidió implementar un reporte estructurado basado en esta categorización, cuya principal utilidad radica en el triaje respiratorio instaurado en nuestra institución.

Palabras clave: COVID-19. Tomografía computarizada. Reporte radiológico.

\section{ABSTRACT}

Introduction: In face of COVID-19 pandemic, CT has been shown to be useful in early stages, since it has been seen that the findings may be present before the onset of symptoms. Recent publications have described the typical and atypical findings on $\mathrm{CT}$, which can be found in patients with COVID-19. Worldwide radiology societies have proposed classification systems to determine the degree of suspicion of COVID-19 based on these findings, as well as proposals for structured reports. Objetive: At the ABC Medical Center, the need has arisen to implement a standardized report that facilitates the integration of the observed findings, reduce interobserver variability, and improve understanding and communication with physicians to enable clinical decision-making. Material and methods: We carried out a review of the classification systems that exist in the literature for the categorization of COVID-19 tomographic findings, based mainly on those that are intended to direct the treatment of suspected cases. Results: The consistency of the findings observed by radiologists from different nationalities and experience, makes the proposed categorization in the consensus of RSNA, ACR and STR, easy to apply and reproducible. Conclusions: We decided to implement a structured radiological report, which main utility is found in the respiratory triage that is established in our institution.

Key words: COVID-19. Computed tomography. Radiological report.

\section{INTRODUCCIÓN}

En diciembre de 2019, la Organización Mundial de la Salud (OMS) fue notificada por primera vez acerca de un brote de neumonía viral de origen desconocido en la ciudad de Wuhan, China. La enfermedad causada por este nuevo virus ha sido denominada enfermedad por coronavirus 2019 (COVID-19) ${ }^{1}$. COVID-19 es ocasionada por un patógeno (Coronavirus tipo 2 del síndrome respiratorio agudo grave [SARS-CoV-2]) que no había sido identificado previamente en humanos ${ }^{2}$. Esta enfermedad ha tenido una rápida propagación a nivel mundial, por lo que el 11 de marzo del 2020 fue declarada pandemia por la $\mathrm{OMS}^{3}$.

Actualmente, la tomografía computarizada (TC) es uno de los métodos de imagen de elección para la evaluación de infecciones pulmonares ${ }^{4}$. Ante la pandemia de COVID-19, la TC ha mostrado ser muy útil en estadios tempranos en los que la radiografía de tórax tiene poca utilidad diagnóstica, ya que se ha visto que los hallazgos en la tomografía pueden estar presentes incluso antes del inicio de los síntomas ${ }^{5}$.

La reacción en cadena de polimerasa en tiempo real (RT-PCR) es la prueba de laboratorio 
ideal para el diagnóstico de virus causantes de enfermedades respiratorias. Durante la fase de transmisión acelerada de la pandemia de COVID-19, el RT-PCR ha sido el principal estudio diagnóstico empleado ${ }^{6}$. Sin embargo, es una prueba que debe interpretarse con cautela, ya que se ha demostrado que el valor predictivo positivo es de tan solo el $60 \%$ en fases iniciales de la enfermedad y, además, se obtienen resultados en al menos 72 horas ${ }^{7}$. Por tal motivo, se ha estudiado el valor diagnóstico y consistencia entre los hallazgos de TC y la positividad de la prueba RT-PCR. Dichos estudios han demostrado una sensibilidad de hasta el 97\% para la TC con hallazgos característicos y la prueba de RT-PCR positiva ${ }^{7}$. No obstante, la TC no deberá considerarse como método de tamizaje para la neumonía en COVID-196,7, debido a su bajo valor predictivo negativo ${ }^{8,9}$.

Publicaciones recientes han descrito los hallazgos típicos y atípicos en la TC, que podemos encontrar en pacientes con COVID-19, así como la evolución de los mismos a lo largo del tiempo ${ }^{5,10,11}$. Diversas sociedades de radiología a nivel internacional han propuesto sistemas de clasificación para determinar el grado de sospecha de COVID-19 con base en los hallazgos por TC, así como propuestas de informes estructurados ${ }^{12-15}$.

En el Centro Médico ABC ha incrementado el número de tomografías solicitadas para pacientes catalogados como casos sospechosos, por lo que surge la necesidad de implementar un reporte estandarizado que facilite la integración de los hallazgos observados, disminuya la variabilidad interobservador y mejore la comprensión y comunicación con los médicos tratantes para permitir la toma de decisiones clínicas oportunas.

\section{REPORTES PROPUESTOS PARA REALIZACIÓN DEL REPORTE RADIOLÓGICO}

Realizamos una revisión de los sistemas de clasificación que existen en la literatura para la categorización de hallazgos tomográficos de COVID-1916. Nuestra revisión se basó principalmente en las clasificaciones que pretenden dirigir el tratamiento de los casos sospechosos. La principal utilidad de nuestro reporte radiológico se encuentra en el triaje respiratorio que se instauró en el Centro Médico $\mathrm{ABC}$ desde las últimas semanas de febrero, ya que el hospital fue dividido desde el inicio en un área COVID y otra no COVID. El principal objetivo de la tomografía fue, por un lado, diagnosticar a pacientes que sí tienen hallazgos característicos o sugestivos, con el objetivo de aislarlos mientras se espera el resultado de la prueba definitiva de RT-PCR y, por otro lado, que aquellos pacientes negativos pudieran ingresar al área no COVID con un riesgo relativamente bajo de contagio, en espera del resultado de RT-PCR.

Se han propuesto varias clasificaciones. Jin, et al. ${ }^{17}$ describió los hallazgos característicos de tomografía en 5 estadios: ultratemprana, temprana, progresión rápida, consolidación y resolución. Sin embargo, esta clasificación funciona para separar a pacientes diagnosticados y confirmados con COVID-19, por lo que no resultaba útil para cumplir nuestro objetivo principal.

La clasificación CO-RADS es un sistema de categorización estandarizado para pacientes con sospecha de infección por SARS-CoV-2, desarrollado para un entorno de prevalencia moderada a alta de la enfermedad. Este es un 
sistema de clasificación propuesto por radiólogos en los Países Bajos y que todavía está en progreso ${ }^{12}$. Dicha clasificación propone 6 categorías que dependen de los hallazgos y el grado de sospecha, como mencionamos a continuación: 1) no sospechoso, 2) levemente sospechoso, 3) moderadamente sospechoso, 4) altamente sospechoso, 5) muy altamente sospechoso (hallazgos típicos), y 6) independientemente de los hallazgos por tomografía, contar con una prueba RT-PCR positiva.

Al realizar un análisis comparativo con otros métodos, nos dimos cuenta que al ser una clasificación que está en progreso y que tiene alta variabilidad interobservador no es la ideal para emplear en el área de triaje respiratorio. Los propios autores de esta propuesta mencionan que es una clasificación que aún debe estudiarse para poder emplearse en la práctica, y por tal motivo no ha sido utilizada a nivel mundial.

Recientemente, en marzo de 2020, se publicó un consenso propuesto por la Sociedad Radiológica de Norteamérica (RSNA), el Colegio Americano de Radiología (ACR) y la Sociedad Torácica de Radiología (STR) para la realización de un reporte radiológico con base en los hallazgos tomográficos atribuibles a COVID-19. El objetivo de este consenso fue orientar a los radiólogos en el informe de hallazgos en TC de los pacientes con sospecha de neumonía en COVID-19, así como aquellos que se descubren de manera incidental ${ }^{9}$. Al ser un consenso de expertos, sabemos que tiene un bajo nivel de evidencia científica. Sin embargo, al ser un consenso propuesto por las principales sociedades de radiología, sabíamos que podíamos utilizarla con ciertas reservas $\mathrm{y}$ eventualmente podríamos realizar un estudio para conocer el impacto de esta categorización en los diferentes desenlaces. Antes de emplearlo como un reporte oficial por nuestro servicio, hicimos una revisión de los estudios sugeridos en el mismo artículo y de otros estudios realizados desde el inicio de la pandemia respecto a la variabilidad interobservador.

Bai, et al. ${ }^{18}$ realizaron un estudio en el que incluyeron a 219 estudios de tomografía de pacientes sospechosos de padecer COVID-19. Se realizó una comparación entre los hallazgos y conclusiones reportados por tres radiólogos de origen chino y cuatro de origen americano. Se identificó que la tomografía es altamente específica para la identificación de pacientes con COVID-19 y que los principales hallazgos de esta neumonía (vidrio esmerilado y distribución periférica) son identificados por la mayoría de los radiólogos.

Existe otro estudio, llevado a cabo por Salehi, et al. $^{5}$, en el que se realizó una revisión sistemática de estudios que mencionaban los hallazgos más característicos en COVID-19. Se incluyeron los estudios de 919 pacientes en los cuales los hallazgos más consistentes fueron presencia de vidrio esmerilado, distribución bilateral, periférica y de predominio posterior.

La consistencia de los hallazgos observados por radiólogos de distintas nacionalidades y experiencia académica, demostrada en estos y otros estudios, hace que la categorización propuesta en el consenso de la RSNA, ACR y STR sea fácil de aplicar y teóricamente pueda reproducirse.

Por tal motivo, pensamos que la aplicación del consenso puede ser reproducible en nuestra propia institución. 
Previo consenso entre los radiólogos del Servicio de Tomografía del Centro Médico ABC se decidió implementar este reporte radiológico para cumplir con nuestro objetivo principal.

\section{ESTRUCTURA DEL REPORTE}

\section{El consenso propone cuatro categorías para} reportar los hallazgos por TC en pacientes catalogados como caso sospechoso de COVID-19. Cada categoría menciona los hallazgos por imagen que podemos encontrar con base en la literatura actual. Asimismo, se propone un lenguaje estandarizado para la conclusión del informe tomográfico:

1. Apariencia típica: son aquellos hallazgos que se consideran característicos en pacientes con COVID-19 (Tabla 1). Dichos hallazgos tienen una alta especificidad, sin embargo, se deben considerar como diagnóstico diferencial la neumonía por influenza y la neumonía organizada, ya que pueden presentar un patrón similar.

2. Apariencia indeterminada: hallazgos que no son típicos en la neumonía COVID-19, y que, al no ser específicos, pueden encontrarse en una amplia variedad de enfermedades (Tabla 2). Por ejemplo, una zona con patrón en vidrio esmerilado sin una distribución típica.

3. Apariencia atípica: hallazgos poco comunes en pacientes con COVID-19 y que normalmente se presentan en otras neumonías de etiología viral (Tabla 3).

4. Negativo para neumonía: ausencia de hallazgos en la tomografía que puedan ser atribuibles a enfermedad infecciosa. Es importante mencionar que la TC puede ser negativa en las primeras etapas de $\mathrm{CO}$ VID-19 (Tabla 4).
TABLA 1. Hallazgos tomográficos y conclusión del reporte radiológico en la categoría Apariencia típica

\begin{tabular}{|c|c|}
\hline Hallazgos tomográficos & Reporte \\
\hline $\begin{array}{l}\text { - Patrón en vidrio esmerilado, } \\
\text { periférico y bilateral (Fig. 1). } \\
\text { - Patrón en vidrio esmerilado } \\
\text { multifocal, morfología } \\
\text { redonda con/sin crazy paving. } \\
\text { - Patrón en vidrio esmerilado } \\
\text { con/sin zonas de consoli- } \\
\text { dación. } \\
\text { - Signo del alo. }\end{array}$ & $\begin{array}{l}\text { - Hallazgos típicos común- } \\
\text { mente reportados en } \\
\text { neumonía COVID-19. } \\
\text { - No se descartan otras } \\
\text { etiologías como neumonía } \\
\text { por influenza o neumonía } \\
\text { organizada, las cuales } \\
\text { pueden presentar patrones } \\
\text { similares. }\end{array}$ \\
\hline
\end{tabular}

TABLA 2. Hallazgos tomográficos y conclusión del reporte radiológico en la categoría Apariencia indeterminada

\begin{tabular}{|l|l|}
\hline Hallazgos tomográficos & Reporte \\
\hline - Ausencia de los hallazgos & - Hallazgos con características \\
típicos. & que pueden verse en \\
- Patrón en vidrio esmerilado, & neumonía COVID-19, no \\
perihiliar o unilateral (Figs. 2 y 3). & específicos, y que pueden \\
- Con/sin zonas de consoli- & tener relación con otros \\
dación sin distribución & procesos infecciosos o \\
específica. & inflamatorios. \\
- Zonas pequeñas de patrón & \\
en vidrio esmerilado (no & \\
redondeadas/no periféricas). &
\end{tabular}

TABLA 3. Hallazgos tomográficos y conclusión del reporte radiológico en la categoría Apariencia atípica

\begin{tabular}{l|l}
\hline Hallazgos tomográficos & Reporte \\
\hline - Ausencia de los hallazgos & - Hallazgos con carac- \\
típicos. & terísticas atípicas o poco \\
- Consolidación lobar aislada con & frecuentes en neumonía \\
patrón en vidrio esmerilado. & por COVID-19. Se deberá \\
- Patrón de "árbol en gemación». & considerar otra posibili- \\
- Cavitación. & dad diagnóstica. \\
- Derrame pleural (Fig. 4). &
\end{tabular}

Para el reporte de hallazgos incidentales, el consenso sugiere informar al médico tratante, y en caso de que el diagnóstico de COVID-19 sea una posibilidad se pueden seguir los lineamientos previamente mencionados. De lo contrario, en caso de que no se pueda establecer la sospecha de COVID-19, los hallazgos 
TABLA 4. Hallazgos tomográficos y conclusión del reporte radiológico en la categoría Negativo para neumonía

\begin{tabular}{|l|l|}
\hline $\begin{array}{l}\text { Hallazgos } \\
\text { tomográficos }\end{array}$ & Reporte \\
\hline $\begin{array}{c}\text { - Ausencia de } \\
\text { hallazgos. }\end{array}$ & $\begin{array}{l}\text { Sin hallazgos de neumonía. (Nota: la } \\
\text { tomografía computarizada puede ser } \\
\text { negativa en las primeras etapas de } \\
\text { COVID-19). }\end{array}$ \\
\hline
\end{tabular}

tomográficos podrán ser reportados como «neumonía de etiología viral».

Nuestro reporte radiológico es sencillo y estructurado (Anexo 1). Incluimos un apartado en el que mencionamos el reporte de dosis y el motivo del estudio, en donde se puede incluir el estatus de la prueba RT-PCR. En el siguiente apartado se mencionan los hallazgos más comúnmente reportados en cada categoría y se da una respuesta dicotómica (sí o no). Para la realización de este último apartado, nos basamos en los hallazgos propuestos por el consenso para cada categoría (zonas en vidrio esmerilado, consolidaciones, signo de halo invertido, engrosamiento septal, árbol en gemación, cavitación, derrame pleural). A continuación existe un apartado en el que se mencionan los hallazgos más relevantes del resto del tórax y lo observado en el hemiabdomen superior. Finalmente, se realiza una conclusión o impresión diagnóstica de acuerdo a lo propuesto en el consenso (Tablas 1 a 4).

\section{NUESTRA EXPERIENCIA EN EL CENTRO MÉDICO ABC}

Posterior a realizar el consenso entre los radiólogos del servicio de tomografía

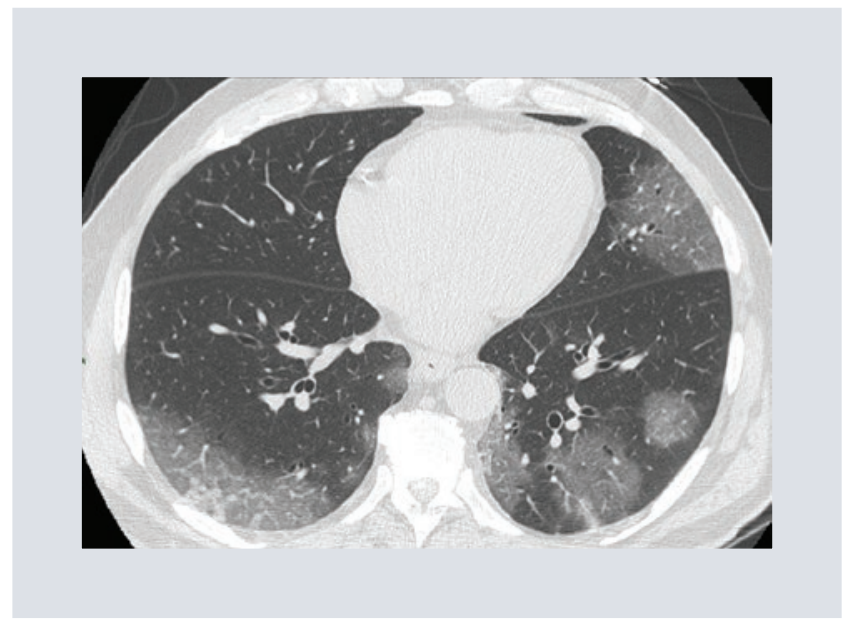

Figura 1. Categoría Apariencia típica. Corte axial de tomografía de tórax en fase simple y con ventana pulmonar, del archivo radiológico del Centro Médico $A B C$. Se identifica el patrón típico de zonas en vidrio esmerilado con distribución periférica y de predominio basal. En el lóbulo inferior derecho se observa una zona que tiende a la consolidación.

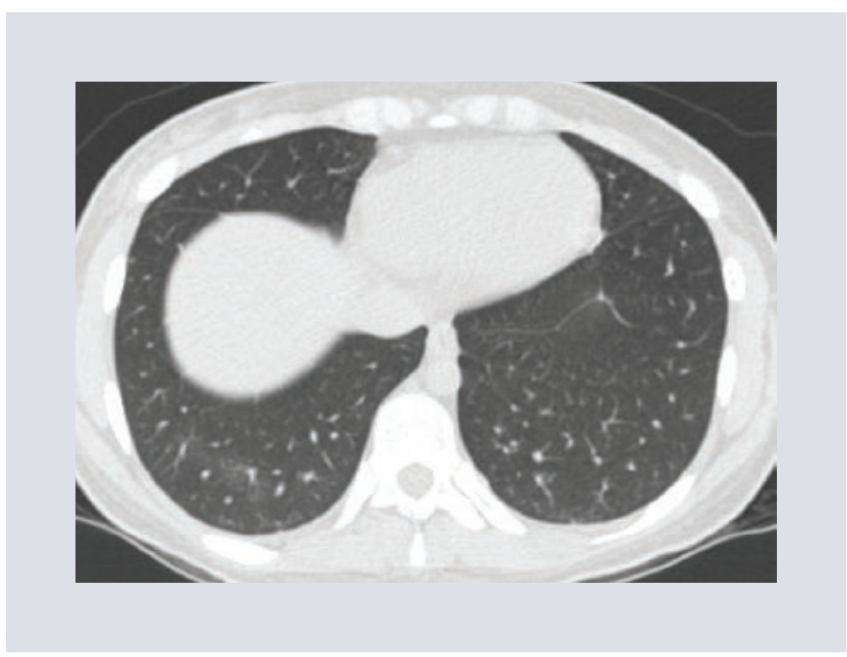

Figura 2. Categoría Apariencia indeterminada. Corte axial de tomografía de tórax en fase simple y con ventana pulmonar, del archivo radiológico del Centro Médico $A B C$. Se identifica una zona mal definida con leve incremento en la densidad en el lóbulo inferior derecho.

comenzamos a catalogar los estudios referidos a TC como pacientes sospechosos para COVID-19. A partir de su implementación en el servicio, ha mejorado la comunicación principalmente con el personal del área de 


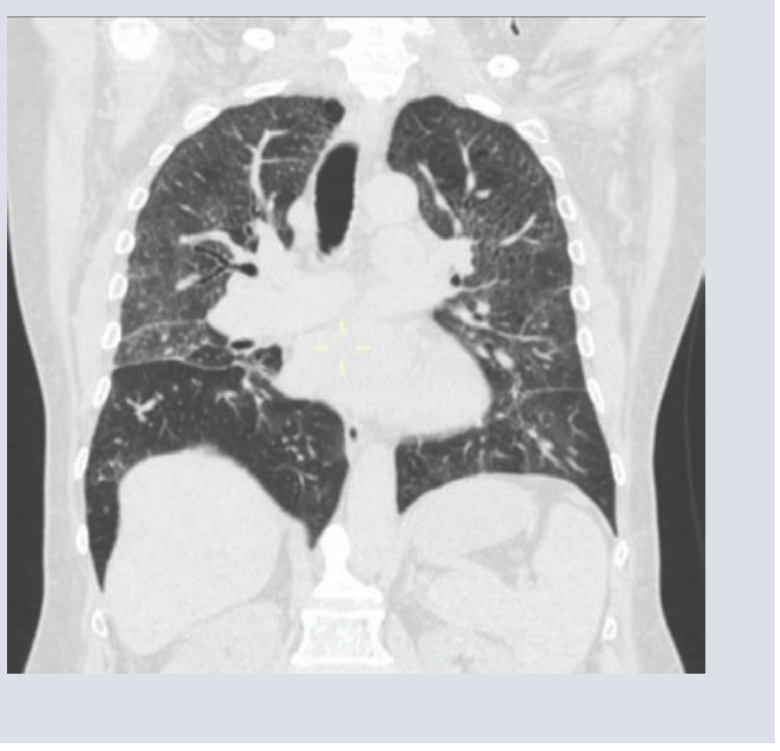

Figura 3. Categoría Apariencia indeterminada. Reconstrucción coronal de tomografía de tórax en fase simple y con ventana pulmonar, del archivo radiológico del Centro Médico ABC. Se identifica enfisema pulmonar centrilobulillar, quistes pulmonares en ambos ápices y bronquiectasias. Sumado a estos hallazgos sugerentes de neumopatía crónica, se identifican zonas mal definidas con incremento en la densidad de forma sutil en todos los lóbulos pulmonares.

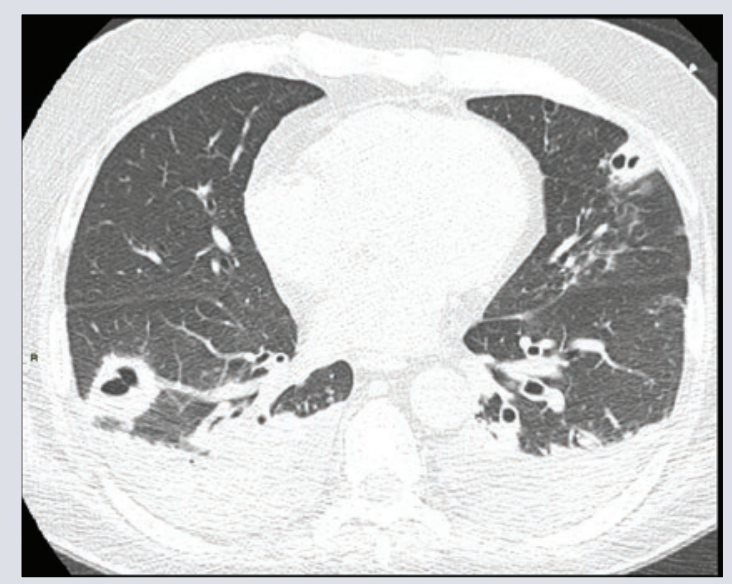

Figura 4. Categoría Apariencia atípica. Corte axial de tomografía de tórax en fase simple y con ventana pulmonar, del archivo radiológico Centro Médico ABC. Se identifican zonas de consolidación pulmonar en segmentos lingulares y lóbulo inferior derecho, que en el centro muestran cavitaciones. Además, existe derrame pleural bilateral que condiciona atelectasias pasivas. Los hallazgos característicos de la apariencia atípica son cavitaciones, derrame pleural y linfadenopatías. Este estudio muestra dos de estos tres hallazgos característicos de la categoría. triaje respiratorio del hospital. La categorización ha homogeneizado el lenguaje con el resto de los servicios hospitalarios, y así, la mayoría del personal involucrado reconoce que aquellos pacientes categorizados como patrón típico e indeterminado pueden ser manejados como pacientes COVID-19 en cuanto a las medidas de protección y el ingreso a las áreas destinadas como COVID en el hospital. Del mismo modo reconocen que los estudios categorizados como atípicos o negativos deben ser tratados como no COVID hasta obtener la prueba definitiva de RT-PCR.

A pesar de esta clasificación y todas sus ventajas, reconocemos y hacemos conocer que tiene sus bemoles. Los hallazgos observados en tomografía deben siempre correlacionarse con los datos clínicos, historia de contacto con casos confirmados y la prueba de RT-PCR.

\section{CONCLUSIONES}

En este escrito demostramos las diferentes clasificaciones que existen para catalogar los hallazgos encontrados en la TC de pacientes sospechosos de padecer COVID-19. Es importante que cada institución emplee su categorización de acuerdo a las necesidades de los servicios y de la población que atienden.

En el Centro Médico ABC hemos empleado la categorización propuesta por la RSNA, ACR y STR, ya que a pesar de ser una categorización con pobre nivel de evidencia para el momento de su publicación, cuenta con cuatro categorías fáciles de entender entre médicos de diferentes especialidades, y 
muestra una variabilidad interobservador baja para la categorización por radiólogos con diferentes niveles de experiencia. Los hallazgos que se han encontrado con mayor frecuencia y con alta sensibilidad para reconocer a los casos típicos de COVID-19 son: presencia de vidrio esmerilado, con distribución periférica y predominio basal.

Hace falta realizar estudios prospectivos que nos permitan evaluar esta maniobra en el hospital con el fin de valorar si su implementación es relevante o no para los diferentes desenlaces de los pacientes. Sin embargo, hasta el momento hemos observado que el uso de este reporte radiológico estructurado por cuatro categorías ha mejorado la comunicación con médicos de otras especialidades $\mathrm{y}$, principalmente, con el personal del triaje respiratorio que se encarga de decidir cuál será el mejor manejo del paciente a su ingreso.

Queremos hacer hincapié en el hecho de que los hallazgos siempre deben correlacionarse con la historia clínica y la prueba de RTPCR. La TC no es un estudio que deba realizarse como tamizaje para descartar COVID-19; sin embargo, es un estudio de imagen que sirve como apoyo diagnóstico. La comunicación clara y frecuente entre los proveedores de salud, incluidos los radiólogos, es imprescindible para mejorar la atención y seguridad de los pacientes durante esta pandemia.

\section{Agradecimientos}

Agradecemos la colaboración y entrega que han tenido cada uno de los médicos y personal de salud que conforman el Centro Médico ABC ante la pandemia COVID-19.

\section{CONFLICTO DE INTERESES}

Los autores declaran no tener conflicto de intereses.

\section{FINANCIAMIENTO}

Los autores declaran no haber recibido financiamiento para la realización de este estudio.

\section{RESPONSABILIDADES ÉTICAS}

Protección de personas y animales. Los autores declaran que para esta investigación no se han realizado experimentos en seres humanos ni en animales.

Confidencialidad de los datos. Los autores declaran que en este artículo no aparecen datos de pacientes.

Derecho a la privacidad y consentimiento informado. Los autores declaran que en este artículo no aparecen datos de pacientes.

\section{BIBLIOGRAFÍA}

1. WHO. Clinical management of severe acute respiratory infection (SARI) when COVID-19 disease is suspected. Guía provisional. Disponible en: https://www.who.int/publications-detail/clinical-management-of-severeacute-respiratory-infection-when-novel-coronavirus-(ncov)-infection-issuspected. Última consulta el 24 de abril del 2020.

2. Zhou P, Yang XL, Wang XG, Hu B, Zhang L, Zhang W, et al. A pneumonia outbreak associated with a new coronavirus of probable bat origin. Nature. 2020;579:270-3.

3. WHO. WHO Director-General's opening remarks at the media briefing on COVID-19 - 11 March 2020. Disponible en: https:/ / www.who.int/dg/speeches / detail/who-director-general-s-opening-remarks-at-the-media-briefing-on-covid-19---11-march-2020. Última consulta el 24 de abril del 2020 
4. Franquet T. Imaging of Pulmonary Viral Pneumonia. Radiology. 2011;260(1):18-39.

5. Salehi S, Abedi A, Balakrishnan S, Gholamrezanezhad A. Coronavirus Disease 2019 (COVID-19): A Systematic Review of Imaging Findings in 919 Patients. Am J Roentgenol. 2020; Mar 14:1-7.

6. Lu G, Wang Q, Gao GF. Bat-to-human: spike features determining. 'host jump' of coronaviruses SARS-CoV, MERS-CoV, and beyond. Trends Microbiol. 2015;23(8):468-78.

7. Yang Y, Yang M, Shen C, et al. Evaluating the accuracy of different respiratory specimens in the laboratory diagnosis and monitoring the viral shedding of 2019-nCoV infections. MedRxiv. 2020 Feb 27. [Online ahead of print].

8. ACR Recommendations for the use of Chest Radiography and Computed Tomography (CT) for Suspected COVID-19 Infection. Disponible en: https: / / www.acr.org/Advocacy-and-Economics / ACR-Position-Statements/Recommendations-for-Chest-Radiography-and-CT-for-SuspectedCOVID19-Infection. Última consulta el 24 de abril del 2020.

9. American Society of Emergency Radiology COVID-19 Position Statement. Disponible en: https://www.aser.org/covid-19-faqs/. Última consulta el 24 de abril del 2020.

10. Geoffrey D. Rubin, Christopher J. Ryerson, Linda B. Haramati, Nicola Sverzellati, Jeffrey P. Kanne, Suhail Raoof, et al. The Role of Chest Imaging in Patient Management during the COVID-19 Pandemic: A Multinational Consensus Statement from the Fleischner Society. Radiology. 2020 Apr 7. [Online ahead of print].

11. Scott S, Kay FU, Abbara S, Bhalla S, Chung JH, Chung M, et al. Radiological Society of North America Expert Consensus Statement on Reporting Chest CT Findings Related to COVID-19. Endorsed by the Society of Thoracic Radiology, the American College of Radiology, and RSNA. Radiology: Cardiothoracic Imaging. 2020;(2):2.
12. Ye Z, Zhang Y, Wang Y, Huang Z, Song B. Chest CT manifestations of new coronavirus disease 2019 (COVID-19): a pictorial review. Eur Radiol. 2020 19 March.

13. Chung M, Bernheim A, Mei X, Zhang N, Huang M, Zeng X, etal. CT Imaging Features of 2019 Novel Coronavirus (2019-nCoV). Radiology. 2020;295(1):202-7.

14. CO-RADS Standardized reporting scheme for Chest CT in patients suspected of COVID-19. Disponible en: https://www.radiologen.nl/system/files / bestanden/documenten/2020-04-10_standaardverslag_ covid-19_co-rads_ppt_pdf_versie_1.0_engels.pdf. Última consulta el 24 de abril del 2020.

15. BSTI. Thoracic Imaging in COVID-19 Infection Guidance for the Reporting Radiologist British Society of Thoracic Imaging Version 2. Disponible en: https: / / www.bsti.org.uk/media/resources / files/BSTI_COVID-19_ Radiology_Guidance_version_2_16.03.20.pdf. Última consulta el 24 de abril del 2020.

16. SERAM. Guía básica de indicaciones de pruebas de imagen en la infección COVID-19. Disponible en: https://www.seram.es/images/site/Recomendaciones_imagen_SERAM_COVID_19.pdf. Última consulta 24 de abril del 2020.

17. Jin YH, Cai L, Cheng ZS, Cheng ZS, Cheng H, Deng T,et al.; Zhongnan Hospital of Wuhan University Novel Coronavirus Management and Research Team; Evidence-Based Medicine Chapter of China International Exchange and Promotive Association for Medical and Health Care (CPAM). A rapid advice guide- line for the diagnosis and treatment of 2019 novel coronavirus (2019-nCoV) infected pneumonia (standard version). Mil Med Res. 2020;7:4.

18. Bai HX, Hsieh B, Xiong Z, Halsey K, Choi JW, Linh TM, et al. Performance of radiologists in differentiating COVID-19 from viral pneumonia on chest CT. Radiology. 2020 Mar 10. [Online ahead of print]. 


\section{Anexo 1. Propuesta de reporte estructurado}

Tomografía simple de tórax

Técnica:

Se realiza adquisición con modalidad helicoidal, abarcando desde el opérculo torácico al abdomen superior, en fase simple.

Reporte de dosis:

Motivo del estudio: Sospecha de COVID-19.

Estatus prueba RT-PCR:

Descripción:

- Opacidades en vidrio despulido: No/Sí (especificar segmentos afectados).

- Consolidaciones asociadas a vidrio despulido: No/Sí (especificar segmentos afectados).

- Zonas con «halo invertido»: No/Sí (especificar segmentos afectados).

- Engrosamiento septal interlobulillar asociado a vidrio despulido: No/Sí (especificar segmentos afectados).

- Hallazgos en el resto del tórax:

- Describir otros hallazgos.

Impresión diagnóstica:

a) Apariencia típica: Hallazgos típicos comúnmente reportados en COVID-19. No se descartan otras etiologías como neumonía por influenza o neumonía organizada, las cuales pueden presentar patrones similares.

b) Apariencia indeterminada: Hallazgos con características que pueden verse en COVID-19, no específicos, y que pueden tener relación con otros procesos infecciosos o inflamatorios.

c) Apariencia atípica: Hallazgos con características atípicas o poco frecuentes en COVID -19. Se deberá considerar otra posibilidad diagnóstica.

d) Negativo para neumonía: Sin hallazgos de neumonía. (Nota: la TC puede ser negativa en las primeras etapas de COVID-19).

COVID-19: enfermedad por coronavirus 2019; RT-PCR: reacción en cadena de polimerasa en tiempo real. 